\title{
V015 INITIAL ANALYSIS OF STABILITY OF PRESTACK VELOCITY INVERSION OF SEISMIC DATA
}

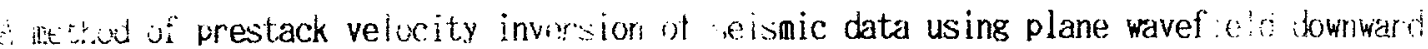
continuation equation has been proposed oy HANGi 1900,1993$]$. Some inversion veicx-ity profiles obtained from prestack marine data have been published in the same paper IWANG, 1990,1993j. However, stability, a key problem with the inversion method (recurent, stripping), has not been discussed. In this note, an initial analys is of the stability problem is introduced.

In brief, a theoretical background of the inversion method is a formulation for downward continuation of plane wavefield in layered medium as the following:

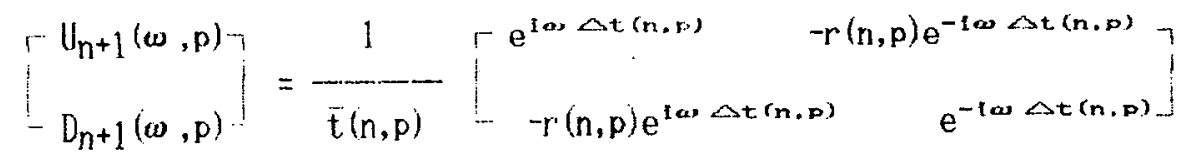

$$
\begin{aligned}
& \times\left[\begin{array}{l}
U_{n}(\omega, p) \\
D_{n 2}(\omega, p)
\end{array}\right]
\end{aligned}
$$

where $\omega$ is a circular frequency, $i=\sqrt{-1}, p=\sin (j(z)) / v(z)$, is a Snell character of plane wavefield, which is constant during propagation in the layered medium, $j(z)$ is an incident angle, $v(z)$ is a velocity function depending on depth, which will be obtained sing the inversion method, and

$$
\begin{aligned}
& r(n, p)=\left(I_{n+1}(p)-I_{n}(p)\right) /\left(I_{n+1}(p)+I_{n}(p)\right) \\
& \bar{t}(n, p)=2 * I_{n+1}(p) /\left(I_{n+1}(p)+I_{n}(p)\right) \\
& I_{n}(p)=v_{n} * \rho_{n} / 1-p^{2} * v_{n}^{2} \\
& \Delta t(n, p)=a_{n} * \sqrt{1-p^{2} * v_{n}^{2} / v_{n}}
\end{aligned}
$$

$\rho_{n}$ is the density of the $n$-th layer, $d_{n}$ is the thickness of the $n$-th layer, $\|_{n}(\omega, p)$ is the up-coming wavefield at top of the layer, $D_{n}(\omega, p)$ is the down-going avefield at top of the layer. If denote $U_{n}^{\prime}(\omega, n)$ and $D_{n}^{\prime}(\omega, p)$ as wavefield at bitom of the layer, according to Claerbort principle, we obtain

$$
r(n, p)=\operatorname{Re} \frac{U_{n}^{\prime}(\omega, p)}{D_{n}^{\prime}(\omega, p)}
$$

Sirce computational steps of the velocity inversion method based on Equ (1) and (2) have been described by Wang $[1990,1993]$, we do not repeat those steps in this note. That, algorithm is complex, so the stability analysis is complex too. The initial analysis is just focused on the Equ (1). For simplicify, Equ (1) can be rewrittern as

$$
x_{n+1}=A_{n} * x_{n}
$$
where

$$
x_{n}=\left[\begin{array}{l}
U_{n}(\omega, p) \\
D_{n}(\omega, p)
\end{array}\right] \quad h_{n}=\left[\begin{array}{ll}
\mathrm{e}^{i \omega \Delta \Delta(n, p)} & -r(n, p) \mathrm{e}^{-i \omega \Delta t(n, p)} \\
-r(n, p) \mathrm{e}^{i \omega \Delta t(r, p)} & \mathrm{e}^{-i \omega \Delta t(n, p)}
\end{array}\right]
$$

Dasn on matrix theory, the stability analysis of Equ (1) or (3) is to discuss the error is, ff ter using $n$ steps of Equ (1) or (3) when the $x_{1}$ has error $\Delta x_{1}$. Easely obtained: 
so that

$$
\Delta \mathrm{x}_{n+1}=\mathrm{A}_{n} \cdot \mathrm{A}_{\mathrm{n}-1} \ldots . . \mathrm{A}_{1} \Delta \mathrm{x}_{1}
$$

and

$$
\text { i\| } \Delta x_{n+1}\|\leqslant\| A_{n}\|\| A_{n-1}\|\ldots . .\| A_{1}\|\| \Delta x_{1} \|
$$

$\left\|A_{n}\right\| \leqslant \max \left(1 \lambda{ }_{n}^{1} 1,1 \lambda \sum_{n}^{2} 1\right)$

is a norm of the matrix $A_{n},\left\|\Delta x_{n}\right\|$ is a norm of vector $\Delta x_{n}$. where $\lambda n_{n}^{1,2}$ are two eigenvalues of the matrix An. After simple operation, obtain

$$
\lambda n_{n}^{1} \cdot 2=\cos \omega \Delta t(n, p) \pm \sqrt{\cos ^{2} \omega \Delta t(n, p)-\left(1-r^{2}(n, p)\right)}
$$

and then procceed to obtain an estimation of the norm of the matrix An:

$$
\left\|A_{n}\right\|<1 \quad \text { when } 1 \cos \omega \Delta t(n, p) 1<1-r^{2}(n, p) / 2
$$

Otherwise,

$$
\begin{aligned}
& 1 \leqslant\left\|A_{n}\right\| \leqslant \sqrt{I_{n+1}(p) / I_{n}(p)} \text { when } I_{n+1}(p)>I_{n}(p) \\
& 1 \leqslant\left\|A_{n}\right\| \leqslant \sqrt{I_{n}(p) / I_{n+1}(p)} \text { when } I_{n}(p)>I_{n+1}(p)
\end{aligned}
$$

Therefore, a conclusion of the stability analysis of Equ (1) or (3) can be shown as:

Def inition: if the impedence function I (p) of medium to plane wavef ield with Snell character $\mathrm{p}$ has $\mathrm{K}$ local maximun values $\mathrm{Iu}(\mathrm{k})(\mathrm{k}=1,2, \ldots, \mathrm{K})$ and $\mathrm{L}$ local minimun values Iv (l) $(1=1,2, \ldots, L)$, then the complex of structure of impedence function can be defined as

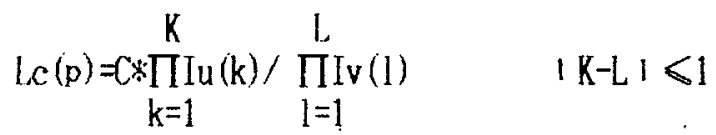

where $\mathrm{C}$ depends on two bourdary values of impendence. Hence, we have

Theorem: If the complex of structure of the impedence function is finite, then for any $n$, there are $\left\|\Delta x_{n}\right\| \leqslant B\left\|\Delta x_{1}\right\|$, where $B \leqslant L c(p)$.

Obviously, if Equ (5) is satisfied for all of $n$, the $\left\|\Delta x_{n}\right\|<\left\|\Delta x_{1}\right\|$, it may be said that Equ (1) or (3) is strictly stable; if Equ (5) is not satisf ied for any $n$, the $B=L c(p), \| \Delta x_{n} !$ is finite too, it may be said that Equ (1) or (3) is weakly stable. When $I(p)$ or $L c(p)$ is infinite, Equ (1) or (3) may be not stable. At this time, pv $(z)=1$, or, $j(z)=\pi / 2$. Therefore, this velocity inversion of prestack seismic data can be operated only on precritical data.

In addition, this inversion method just needs two plane wavef ield data sets with same frequrency and different Snell characters on the theory; When apply the inversion method to real data, the multi-frequrency and multi-Snell-character plane wavefield data are needed to obtain more accurate velocity.

\section{REFERENCE}

[1] Tianxi WANG (1990), Prestack inversion of seismic data with plane-layer medium and point source by wavefield downward continuation, SEC 60th Annual Meeting, SP20.

[2] Tianxi WANG (1993), Prestack velocity inversion of seismic data using plane wavefield downward continuation, Chinese Journal of Geophysics, vol 36 , № 3, pp 405-413, by Allerton Press, INC. 